\title{
EC02009 Hot Topic Abstracts
}

\section{7th European Congress on Obesity (ECO2009)}

Amsterdam, the Netherlands

May 6-9, 2009 


\section{HT:PO.01 \\ The associations between skipping meals and overweight among Norwegian adolecents}

\author{
Vik F, Strømmen T, Vik G, Bere $E$ \\ University of Agder, Kristiansand, Norway
}

Introduction: Eating meals, and thereby snacking less, might prevent excess weight gain. The purpose of the present study was to assess the relationships between skipping meals and weight status.

Methods: A total of 2,870 adolescents (mean age: 15.5 years) at 33 schools completed questionnaires in May 2005 (project FVMM). Questions included whether they ate breakfast, lunch, dinner and supper yesterday. Sex, height, weight, education plans (as an indicator of SES), intake of fruits and vegetables (FV), consumption of unhealthy snacks, TV/PC watching and physical activity level were also measured.

Results: A total of $21 \%$ skipped breakfast, $15 \%$ skipped lunch, $8 \%$ skipped dinner and $28 \%$ skipped supper. Those skipping breakfast and supper were more often overweight than those not skipping those meals, respectively $16.2 \%$ vs. $11.3 \%(\mathrm{p}=0.03)$ and $15.4 \%$ vs. $11.1 \%(\mathrm{p}=0.03)$ were overweight. The proportion overweight related to number of meals eaten were: $10 \%(0-1$ meals, $n=107), 18 \%(2$ meals, $n=399), 14 \%(3$ meals, $\mathrm{n}=925)$ and $10 \%(4$ meals, $\mathrm{n}=1402), \mathrm{p} \leq 0.001$. Skipping any meal was related to a lower FV intake, a higher consumption of unhealthy snacks, more TV/PC watching and to a lower physical activity level. In a logistic regression analysis, adjusting for all variables mentioned, OR for being overweight was 1.2 (95\% CI 0.5-2.7), 2.0 (95\% CI 1.3-3.0) and 1.7 (95\% CI 1.2-2.3), respectively for eating $0-1,2$ and 3 meals compared to 4 meals.

Conclusion: Skipping one or two meals was significantly related to being overweight, also after adjusting for sex, education plans, eating and activity behaviours.

Conflict of Interest: None Disclosed. Funding: Research relating to this abstract was funded by the Norwegian Research Council and the seventh framework programme of the European Union.

\section{HT:PO.02}

\section{Development of instruments to assess the obesogenicity} of urban environments in Europe

\author{
Pomerleau $J^{1}$, Knai $C^{1}$, Foster $C^{2}$, Robertson $A^{3}, M c K e e ~ M^{1}$, \\ Darmon $N^{4}$, Derflerova Brázdová $Z^{5}$, \\ Filipovic Hadziomeragic $A^{6}$, Pekcan $G^{7}$, Pudule $I^{7}$, Rutter $H^{8}$, \\ Brunner $E^{9}$ on behalf of the EURO-PREVOB Consortium \\ 'London School of Hygiene and Tropical Medicine, London, UK \\ ${ }^{2}$ University of Oxford, Oxford, UK \\ ${ }^{3}$ SUHR'S University College, Copenhagen, Denmark \\ ${ }^{4}$ UMR INRA 1260 / INSERM 476 / Universités Aix -Marseille I and II, \\ Marseille, France \\ ${ }^{5}$ Masaryk University, Brno, Czech Republic \\ ${ }^{6}$ Institute of Public Health of the Federation of Bosnia and \\ Herzegovina, Sarajevo, Bosnia and Herzegovina \\ ${ }^{7}$ Hacettepe University, Ankara, Turkey \\ ${ }^{8}$ Public Health Agency, Riga, Latvia \\ ${ }^{9}$ South East Public Health Observatory, Oxford, UK \\ ${ }^{10}$ University College London, London, UK
}

Introduction: While there is a growing body of research focusing on the influence of 'obesogenic' environments on obesity, there is a lack of instruments that can measure various aspects of the environment in different contexts. The EURO-PREVOB project thus developed and tested a new set of instruments designed to capture contextual differences, within and between countries, in environmental indicators relevant to obesity in Europe.

Methods: The instruments examine, in urban areas of different socioeconomic levels, the food environment (availability of shops selling foods, cost of indicative food items, advertising on children's television, cost and marketing of fast-food items) and the built environment (availability and quality of cycle lanes, parks, public transport stops, road crossings, pavements). The instruments were piloted in five cities (Ankara, Turkey; Brno, Czech Republic; Marseille, France; Riga, Latvia; Sarajevo, Bosnia and Herzegovina).

Results: The development of the instruments faced many practical and methodological challenges, including access to high quality maps, authorised data collection in grocery stores, difficulties in sampling areas of varying socioeconomic levels, and differences in the meaning of contextual variables (e.g. choice of indicative foods, quality criteria for aspects of the built environment). The methods were generally relevant to all countries but variations were observed in their applicability. Differences were also noted in many markers of obesogenicy.

Conclusions: EURO-PREVOB has piloted a novel method to assess obesogenicity in diverse settings. The tool has to implemented on a larger scale to generate policy-relevant observations about key modifiable factors linked with child and adult obesity rates,

Conflict of Interest: None. Funding: Research relating to this abstract was funded by the European Commission Sixth Framework Programme (Project no. 044291). This abstract reflects only its authors' views.

\section{HT:PO.03}

\section{Gastric bypass is superior in quality of life compared to gastric banding independent from weight loss}

\section{Miller $K^{1}$, Parzer $S^{2}$,Ardelt-Gattinger $E^{2}$}

${ }^{1}$ Krankenhaus Hallein, Austria,

${ }^{2}$ University Salzburg, Psychological Institute, Austria

Introduction: In bariatric surgery studies show that a better quality of life is correlated to increased weight loss. The question remained which type of surgery is superior in quality of life independently from weight loss.

Methods: In our study we recruited 250 bariatric patients, operated between 2003 and 2005 (196 women/ 54 men) at the mean age of 39.85 years $(\mathrm{SD}=11.65)$ and with a mean BMI of $44.78 \mathrm{~kg} / \mathrm{m}^{2}(\mathrm{SD}=7.04)$. Patients eligible for investigation were 78 Patients with Laparoscopic Gastric Bypass and 172 Patients with Adjustable Gastric Banding (AGB). The patients were reviewed in the interval of 3, 6, 12,24,36, 48 and 63 months after operation. The Bariatric Analysis and Reporting Outcome System (BAROS) was used for the quality of life investigation which has been international established for obesity surgery outcomes.

Results: With a minimum of 3 years follow-up our patients showed a mean BMI of $28.8 \mathrm{~kg} / \mathrm{m}^{2}(\mathrm{SD}=6.65)$. The statistical analysis (linear regression) showed a positive correlation between quality of life and weight loss, depending on operation method. Additionally we used a partial correlation to rule out the influence of weight loss and remarked a significant result $(\mathrm{r}=.234, \mathrm{p}=.000)$. With a t-test it could be demonstrated, that patients with a Laparoscopic Gastric Bypass observed a significant different quality of life, than patients with an AGB independent from weight loss $(t(123)=3,477, p=.001)$.

Conclusion: Independently of the amount of weight loss, gastric bypass is the surgical procedure, which leads to a significant higher quality of life. Conflict of Interest: No payment received. Investigator initiated study. Funding: No funding relating to this abstract and study was funded.

\section{KARGER}

Fax +497614520714

Information@Karger.de

www.karger.com (c) 2010 S. Karger GmbH, Freiburg

Accessible online at:

www.karger.com/ofa 


\section{HT:PO.04}

Identifying the energy gap in the German population using data from national health surveys between 1985 and 2005

\section{Stroebele $\mathrm{N}^{1}$, Willich $S N^{1}$, Hill JO}

${ }^{1}$ Institute for Social Medicine, Epidemiology, and Health Economics, Charité University Medical Center, Berlin, Germany

${ }^{2}$ University of Colorado Denver, Center for Human Nutrition, Denver USA

Introduction: The prevalence of overweight and obesity is increasing in most countries including Germany. Hill et al. introduced the idea of estimating the population-wide energy gap that is likely to be responsible for the epidemic. It was hypothesized that by reducing energy intake by $100 \mathrm{kcal}$ per day, most of the weight gain in the US population could be prevented.

Methods: Using body measurements and self-reported data of nationa health surveys (1985-2003), the energy gap was calculated by estimating the distribution of the rate of weight gain within the German population (25-69 years) and the amount of excess energy storage that would be required to support this population-wide pattern of weight gain.

Results: Men gained on average $0.54 \mathrm{~kg}$ and women $0.77 \mathrm{~kg}$ per year Assuming that each $\mathrm{kg}$ of weight gained represents $7,700 \mathrm{kcal}$, the estimated energy accumulation is about $11.4 \mathrm{kcal} /$ day in men and $16.2 \mathrm{kcal} /$ day in women. With an estimation of energy efficiency of $50 \%$, the yearly weight gain could be prevented with less than a $100 \mathrm{kcal}$ reduction of energy intake or increase of energy expenditure per day.

Conclusion: Although this calculation is based on theoretical estimations and empirical results are needed, further weight gain can likely be pre vented using a small changes approach that emphasizes the importance of making small changes in physical activity as well as food intake. The public and the private sectors in Germany need to work together to create an environment that encourages people to make small changes towards a healthier lifestyle.

Conflict of Interest: None disclosed. Funding: No funding.

\section{HT:PO.05}

\section{Body mass index of Finnish young men entering military service is associated with educational level and snacking-type eating habits}

\section{Bingham $C M L^{1}$, Jallinoja $P^{1}$, Absetz $P^{1}$, Lahti-Koski $M^{2}$,} Paturi $M^{1}$, Pihlajamäki $H^{3}$, Sahi $T^{4}$, Uutela $A^{1}$

${ }^{1}$ National Institute for Health and Welfare, Helsinki, Finland

${ }^{2}$ Finnish Heart Association, Helsinki, Finland

${ }^{3}$ Centre of Military Medicine, Lahti, Finland

${ }^{4}$ Department of Public Health, University of Helsinki, Helsinki, Finland

Introduction: Obesity is increasing among young men but little is known about underlying factors and their contributions to the phenomenon. As Finnish men have liability to military service and over $80 \%$ of each age category complies with it, the military setting offers feasible circumstances to reach this population group. Relations between body mass index (BMI), educational level and eating habits were examined in this study. Methods: In 2007, data on weight, height, education and eating habits of 2,905 17-21-year-old men $(\mathrm{n}=2,905)$ entering military service were collected by self-administrated questionnaire. Two indexes (1) core food index (CFI) and (2) extra food index (EFI) were formed to describe daily and redundant snacking-type eating, respectively. The significances of educational level, CFI and EFI on BMI were analysed by linear regression model.

Results: Based on self-reported BMI, 24.4\% of men were overweigh $(\mathrm{BMI}=25.0-29.9)$ and $7.8 \%$ were obese $(\mathrm{BMI} \geq 30)$. BMI was significantly lower $(\mathrm{p}>0.0001)$ for those having completed upper secondary school compared to lower basic educational levels. BMI was associated with educational level $(\mathrm{p}<0.0001)$, CFI $(p=0.048)$ and EFI $(p<0.0001)$ However, in multivariate analysis, educational level $(\mathrm{p}<0.0001)$ and EFI $(\mathrm{p}<0.0001)$ explained BMI but CFI did not.
Conclusions: These results indicate that already in young adulthood the influence of daily eating habits on BMI is explained by the level of basic education. Redundant snacking-type eating, however, is associated with BMI irrespective of educational level.

Conflict of Interest: None disclosed. Funding: Research relating to this abstract is funded by the Academy of Finland.

\section{HT: PO. 06}

\section{What effect does social class have on weight control in mid-aged women? Evidence from the Australian Longitudinal Study on Women's Health}

\author{
Williams L, Germov J, Young A \\ University of Newcastle, Newcastle, Australia
}

Introduction: The associations between social class and obesity are relatively well established and members of the working class in developed countries have higher rates of obesity and disease risk factors. Less is known about the relationship between class, weight gain, and the practices used to prevent weight gain. This longitudinal study examined associations between self-defined social class, socio-demographic factors (income, education, occupation, area of residence), weight control practices, and two-year weight change among 11589 mid-aged women (aged 47-52) participating in the Australian Longitudinal Study on Women's Health.

Methods: Frequency of weight control practice was calculated according to self-selected social class (upper, middle, or working class). A multivariate model was developed to test whether mean weight change over a two-year period differed by social class after adjusting for age, education, baseline BMI, and smoking. Statistical analyses were conducted using SAS.

Results: While the entire cohort showed a mean weight gain, women who reported being working class had a significantly higher mean (se) two-year weight gain at $1.27(0.07) \mathrm{kg}$ compared with middle/upper class women at $1.01(0.07) \mathrm{kg}$. Working class women were significantly more likely to use potentially harmful weight control practices $(12.8 \%)$ than upper/middle class women $(8.9 \%)$ (chi-squared test $=30.65, \mathrm{p}<0.0001)$, and were less likely to use exercise to control weight.

Conclusion: This is the first Australian longitudinal evidence that weight control practices and weight gain are related to social class. The findings have implications for targeting weight gain prevention programs to this high-risk group.

Conflict of Interest: None. Funding: Research relating to this abstract was funded by the University of Newcastle.

\section{HT:PO.07}

\section{Waist to height ratio 0.5 as a simple screening tool for central obesity and cardiovascular risk factors}

\section{Ashwell $M^{1}$, Gibson $S^{2}$}

${ }^{1}$ Ashwell Associates ( Europe) Ltd, Ashwell, UK

${ }^{2}$ Sig-Nurture Ltd, Guildford, UK

Introduction: BMI does not distinguish central obesity; waist circumference (WC) requires different cut-offs for men, women and children. Can a single boundary value of waist to height ratio (WHtR) be a global screening indicator for central obesity and cardiovascular (CVD) risk?

Method: National Diet and Nutrition Survey data (2001) were used to examine associations with CVD risk factors in 1,776 adults aged 19 to 64 years.

Results: WHtR was more highly correlated with total, HDL, non-HDL cholesterol and BP than BMI and WC. Classification by BMI (above or below $25 \mathrm{~kg} / \mathrm{m}^{2}$ ) and WHtR (above 0.5 = 'apples' or below 0.5 = 'pears') showed 'apples' had higher levels of all risk factors (higher TC, nonHDL cholesterol , SBP, DBP, but lower HDL cholesterol) than 'pears' who had similar levels of risk factors, whether they were overweight or not. Most interesting, non-overweight 'apples' were at higher risk than 
overweight 'pears'; (non-HDL cholesterol + $0.3 \mathrm{mmol} / \mathrm{l}, \mathrm{SBP}+4 \mathrm{~mm} \mathrm{Hg}$, DBP $+3 \mathrm{~mm} \mathrm{Hg}$. If screened by BMI alone, $17 \%$ of all men and $6 \%$ of all women would be misclassified as without risk

Conclusion: WHtR is a simple, effective, non-invasive, global screening tool for CVD risk factors. We propose a simple public health message 'Keep your waist circumference to less than half your height' (WHtR $<0.5)$.

Funding: Lipton Institute of Tea, part of Unilever plc.

\section{HT:PO.08}

Growth curves in body mass index by birth cohort in Japanese boys and young men: Repeated cross sectional annual nationwide survey over nearly six decades

\section{Funatogawa $I^{1}$, Funatogawa $T^{2}$, Yano $E^{1}$}

${ }^{1}$ Teikyo University School of Medicine, Tokyo, Japan

${ }^{2}$ Chugai Pharmaceutical Co, Ltd, Tokyo, Japan

Introduction: Obesity in childhood has become an important public health concern worldwide, but shapes of growth curves in body mass index (BMI) from childhood to adulthood by birth cohort have not drawn much attention. We previously constructed growth curves of BMI in Japanese girls and young women (Funatogawa et al. BMJ, 337:a802, 2008) and Japanese adults (Funatogawa et al. Int J Epidemiol, 38:83-92, 2009), and demonstrated the different shape by birth cohort. In this study, we produce growth curves in Japanese boys and young men.

Methods: We compared growth curves in Japanese men aged 1-25 among multiple birth cohorts born from 1930s to 1990s based on annual nationwide surveys (national nutrition survey, Japan) carried out from 1948 to 2005 . We further compared the results between men and women. Results: Generally, after 5 years of age, BMI was higher in more recent cohorts of Japanese men. The difference among birth cohorts was most apparent in late childhood (about 11 years), but the difference became smaller in late adolescence (about 18 years). This trend was somewhat different from the results of Japanese women who showed higher BMI in childhood in more recent cohorts, but lower BMI in young adulthood. Conclusion: Monitoring growth curves in BMI for wide age ranges by birth cohort provides better understanding of obesity. A repeated cross sectional annual survey is suitable for this purpose.

Conflict of Interest: None. Funding: Research relating to this abstract was funded by MEXT KAKENHI (20700255).

\section{HT:PO.09}

\section{A life-course approach provides evidence for fruit consumption in adult obesity prevention in a British Birth Cohort}

\section{Chen $Y^{1}$, Mander $A P^{1}$, Hardy $R^{2}$, Stephen $A M^{1}$}

${ }^{1} \mathrm{MRC}$ Human Nutrition Research, Cambridge, UK

${ }^{2} \mathrm{MRC}$ National Survey of Health and Development, London, UK

Introduction: The evidence for fruit consumption in preventing obesity is inconsistent. This study uses a life-course approach to study the role of consistently high intake of fruit in obesity prevention in a British Birth Cohort.

Methods: 5,362 children were selected as a random sample of all babies born between 3rd-9th March 1946 in Britain. Fruit consumption information was measured from $24 \mathrm{~h}$-recall at age 4 , and 5-d diaries at ages 36 , 43 years and was brought together to predict body mass index (BMI) at age 53 with adjustment for gender and childhood social class.

Results: Analysis on 1,482 subjects shows the number of times being in the high fruit consumption category $(\beta=-0.35, \mathrm{p}<0.01)$ and the tracking pattern $(\beta=-0.15, \mathrm{p}<0.01)$ through ages $4-43$ years was significantly associated with BMI $\left(\mathrm{m}^{2} / \mathrm{kg}\right)$ at age 53 .

Conclusion: This study provides evidence for fruit promotion programs in tackling obesity. People can get immediate benefit from 'above-population-average' consumption of fruit at various stages of life. A life-

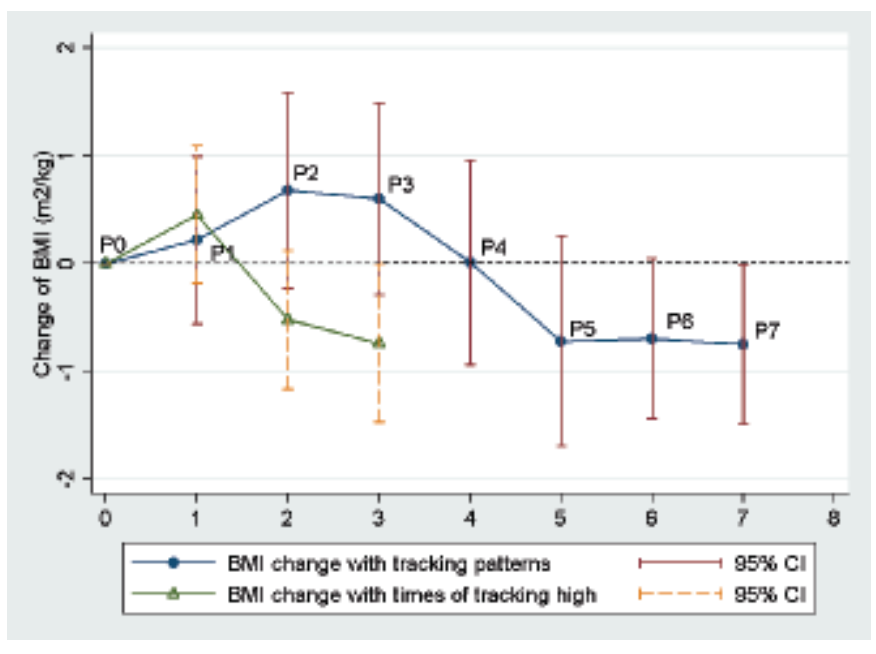

course approach is more powerful than prospective analysis in investigating diet and obesity in populations.

Conflict of Interest: No conflict of interest. Funding: Research relating to this abstract was funded by Cambridge Overseas Trust, Overseas Research Studentship and MRC Human Nutrition Research.

\section{HT:PO.11}

Survival advantages of overweight and obesity in 121,762 maintenance hemodialysis patients in the USA from 2001 to 2006

\section{Streja $E^{1}$, Jing $J^{1}$, Nissenson $A R^{2}$, Kalantar-Zadeh $K^{1}$ \\ 'Harold Simmons Center at Harbor-UCLA, \\ ${ }^{2}$ DaVita, Inc, Torrance and EI Segundo, CA, USA}

Background: Whereas overweight and obesity are associated with poor outcomes in the general population according to most epidemiologic studies, in populations with chronic disease states and high risk of wasting syndrome such as individuals with chronic kidney disease (CKD) or chronic heart failure an 'obesity paradox' or 'reverse epidemiology' has been observed, in that higher height-adjusted body weight is counterintuitively associated with greater survival. It is not clear whether variations of weight or other clinical or laboratory values over time would affect these associations.

Methods: We examined the time-dependent associations in a large national cohort of outpatients with CKD stage 5 (end-stage renal disease) who required maintenance hemodialysis patients to survive. The national cohort consisted of 121,762 patients (from 7/2001 to 6/2006) whose body mass index (BMI) could be calculated based on post-dialysis dry

Survival of 121,762 hemodialysis patients over 5 yrs (7/2001-6/2006)

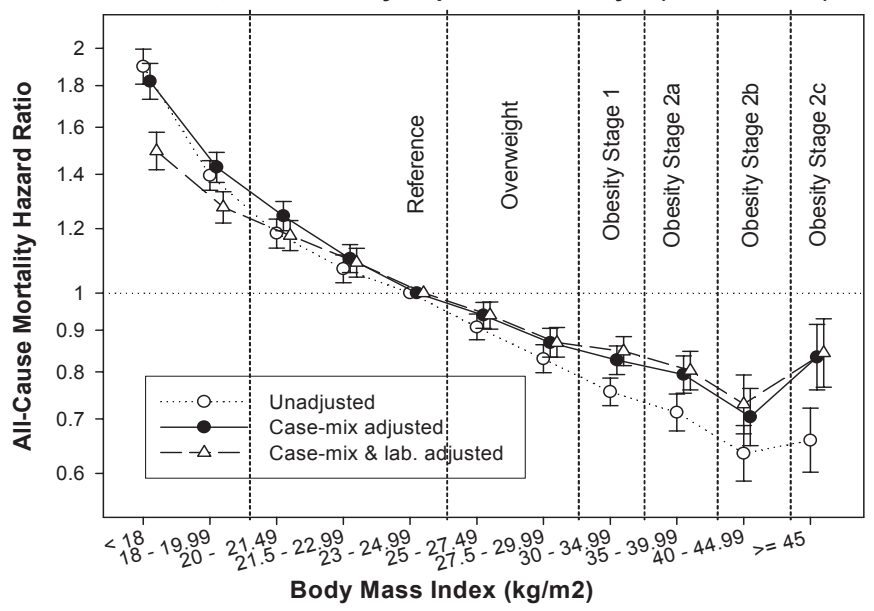


weight divided by height square during each thrice-weekly dialysis treatment, and the values were averaged during each calendar quarter, i.e., up to 39 thrice weekly dry weight values per quarter for up to 5 years or up to death or censorship. Time-dependent Cox model was performed for a -priori selected BMI categories.

Results: Patients were $61.1 \pm 15.6$ years old and included $45 \%$ women, $32 \%$ African Americans, 14\% Hispanics and 43\% diabetics. Higher BM values between 25 and $45 \mathrm{~kg} / \mathrm{m}^{2}$ were incrementally and linearly associated with greater survival, whereas low normal to low BMI values were linked to increased death risk.

Conclusions: Survival advantages of higher BMI values up to $45 \mathrm{~kg} / \mathrm{m}^{2}$ are observed in a large national cohort of American hemodialysis outpatients, even after accounting for changes in clinical and laboratory values over time. The risks vs. benefits of weight reduction in obese hemodialysis patients need to be investigated before it can be recommended.

Conflict of Interest: None disclosed. Funding: National Institutes of Health (NIH) grant R01DK078106 for KKZ

\section{HT:PO.12}

\section{Impact of feeding pattern on body composition: implication of ghrelin}

\section{Verbaeys $I^{1}$, Swennen $Q^{2}$, Willemsen $H^{2}$, Buyse $\mathrm{J}^{2}$, Tolle $\mathrm{V}^{\mathcal{B}}$, Zizarri $P^{3}$, Cokelaere $M^{1}$}

${ }^{1}$ Interdisciplinary Research Centre, Campus Kortrijk, Kortrijk, Belgium ${ }^{2}$ Lab of Livestock Physiology, Immunology and Genetics of Domestic Animals Katholieke Universiteit Leuven, Leuven, Belgium ${ }^{3}$ UMR894 INSERM, Centre de Psychiatrie et Neurosciences, Paris, France

Introduction: Rats with an imposed feeding pattern display a slower growth rate compared to ad libitum fed controls, despite an equal daily calorie intake. Endogenous ghrelin, known to control growth and stimulate adipogenesis, is closely correlated to meal patterns. Therefore, we hypothesized that a chronic imposed feeding pattern might induce changes in ultradian ghrelin rhythm and a consequent modification in body composition.

Methods: During 14 consecutive days, juvenile rats received three meals per day distributed as follows: $20 \%$ of the daily food consumed by ad libitum fed rats at the onset of the dark cycle, $25 \% 3$ hours later and 55\% 6 hours after dark onset). On day 14, variations in plasma ghrelin levels were monitored every 20 minutes during 9 consecutive hours in freely moving animals. DEXA scans were performed in these rats to elucidate their whole body composition. A group, daily injected with ghrelin (100 $\mu \mathrm{g} \mathrm{kg}^{-1}$, ip) for 14 days, was included to explore whether a chronic increase in ghrelin levels in growing rats stimulate adipogenesis.

Results: Compared to ad libitum fed controls, rats with an imposed meal pattern displayed elevated plasma ghrelin levels and a higher fat/lean ratio. Interestingly, rats treated with exogenous ghrelin showed a similar increase in fat mass.

Conclusion: Our results suggest that a forced meal pattern, even without a nutrient/calorie restriction, results in a higher fat/lean ratio. This repartitioning effect, also observed in the ghrelin-group, might be caused by the increased endogenous ghrelin-levels induced by the forced food pattern.

Funding: This work is supported by 'Onderzoeksraad K.U.Leuven' OT 04/32 and FondsWetenschappelijk Onderzoek G. 0519. 05.

\section{HT:PO.13}

Supporting the 'uncoupling-to-survive' theory of aging: a low resting metabolic rate is related to high free-radical scavenging activity of the mitochondria

\author{
Johannsen, $D L^{1}$, Gallagher, $D^{2}$, Ravussin, $E^{1}$, \\ for the Louisiana Healthy Aging Study \\ ${ }^{1}$ Pennington Biomedical Research Center, Baton Rouge, LA, USA \\ ${ }^{2}$ Columbia University, New York, NY, USA
}

Studies have shown that resting metabolic rate (RMR) decreases with age independent of changes in body composition. A low relative RMR has been associated with longevity, but may also be associated with greater reactive oxygen species production ('Uncoupling to Survive' theory of aging). We investigated the determinants of RMR and hypothesized that decreased organ volume would largely explain the reduced RMR in aging. Eleven elderly ( $77 \pm 3 \mathrm{y}, \mathrm{SD} ; 7$ male) and 10 young (27 \pm 4 y; 7 male) volunteers matched for BMI had measures of organ and tissue volume by MRI, fat free mass (FFM) by DXA, and RMR by indirect calorimetry. Muscle tissue was collected by biopsy. Despite less skeletal muscle $(\mathrm{p}=0.03)$, RMR was not significantly lower in elderly compared to young volunteers $(1,331 \pm 173$ vs. $1,477 \pm 309 \mathrm{kcal} / \mathrm{d}$ respectively, $\mathrm{p}=$ $0.19)$. After adjustment of RMR for FFM $(\mathrm{p}<0.001)$ and sex $(\mathrm{p}=0.02)$ $\left(\mathrm{R}^{2}=0.51\right)$, age group had no effect on RMR. Organ volumes (brain, kidney, spleen, liver, and pancreas) did not improve the ability to predict RMR. Interestingly, muscle glutathione peroxidase activity $\left(\mathrm{p}=0.02 ; \mathrm{R}^{2}\right.$ $=0.62$ ), explained an additional $11 \%$ of variance in RMR. Markers of oxidative damage (DNA COMET tail length, muscle protein carbonyls), however, had no effect. Contrary to our hypothesis, organ volume did not explain much variability in RMR. Instead, we found that higher antioxidant activity was associated with lower relative RMR. This novel data supports the 'uncoupling to survive' theory. Our in vivo mitochondrial efficiency data ( $\mathrm{P} / \mathrm{O}$ ratio) will be available at the meeting.

\section{HT:PO.14}

\section{Gluconeogenesis and energy expenditure after a high protein, carbohydrate-free diet}

\section{Veldhorst $M$, Westerterp-Plantenga $M$, Westerterp $K$}

${ }^{1}$ NUTRIM School for Nutrition, Toxicology and Metabolism, Department of Human Biology, Maastricht University Medical Centre+, The Netherlands, and the Top Institute Food and Nutrition, the Netherlands

Background: A high protein diet, especially in the absence of carbohydrates, may stimulate gluconeogenesis (GNG) and this process may be responsible for the increased energy expenditure at such a diet.

Aim: To study whether a high protein, carbohydrate-free diet increases GNG and can explain the increase in energy expenditure.

Methods: Ten healthy male subjects (mean \pm SEM BMI: $23.0 \pm 0.8 \mathrm{~kg} / \mathrm{m}^{2}$, age: $23 \pm 1$ years) received an iso-energetic high protein, carbohydratefree $(\mathrm{H}, 30 / 0 / 70 \mathrm{En} \%$ protein/carbohydrate/fat) or a normal diet $(\mathrm{N}$ $12 / 55 / 33 \mathrm{En} \% \mathrm{P} / \mathrm{CHO} / \mathrm{F}$ ) for one and a half day in a randomized, crossover design in a respiration chamber. Endogenous glucose production (EGP) and fractional GNG were measured using infusion of $\left[6.6-{ }^{2} \mathrm{H}_{2}\right]$ glucose and ingestion of ${ }^{2} \mathrm{H}_{2} \mathrm{O}$; absolute $\mathrm{GNG}$ was calculated by multiplying fractional GNG with EGP. Body glycogen stores were lowered at the start of the intervention with an exhaustive glycogen-lowering exercise test.

Results: EGP was lower in $\mathrm{H}$ than in $\mathrm{N}(181 \pm 9 \mathrm{~g} / \mathrm{d}$ vs. $226 \pm 9 \mathrm{~g} / \mathrm{d}$, p < $0.001)$ whereas fractional GNG $(0.95 \pm 0.04$ vs. $0.64 \pm 0.03, p<0.001)$ and absolute GNG $(171 \pm 10 \mathrm{~g} / \mathrm{d}$ vs. $145 \pm 10 \mathrm{~g} / \mathrm{d}, \mathrm{p}=0.06)$ were higher. The increase in resting metabolic rate (RMR) in $\mathrm{H}$ compared with $\mathrm{N}$ (8.46 $\pm 0.23 \mathrm{MJ} / \mathrm{d}$ vs. $8.12 \pm 0.31 \mathrm{MJ} / \mathrm{d}, \mathrm{p}<0.05)$ was a function of the increase in $\mathrm{GNG}\left(\Delta \mathrm{RMR}=0.0046^{*} \Delta \mathrm{GNG}-0.0021, \mathrm{r}=0.65, \mathrm{R}^{2}=0.42, \mathrm{p}<0.05\right)$. Conclusion: A major part of the increased energy expenditure at a highprotein, carbohydrate-free diet can be explained by increased gluconeogenesis. 


\section{HT:PO.15}

\section{A 12-week exercise programme improves short-term appetite regulation in overweight/obese volunteers}

\author{
Martins $C^{1}$, Kulseng $B^{1}$, Blundell $J E^{2}$ \\ ${ }^{1}$ Norwegian University of Science and Technology, Trondheim, \\ Norway \\ ${ }^{2}$ University of Leeds, Leeds, UK
}

Introduction: Cross-sectional studies have shown a more accurate en ergy compensation in active versus sedentary normal-weight individuals Moreover, a 6-week exercise programme has been shown to improve short-term appetite regulation over a 24-hour period in normal-weight individuals. This study aims to investigate the effects of a 12 -week exercise programme ( 5 times/week, $75 \%$ maxHR) on appetite regulation in overweight/obese sedentary volunteers.

Methods: Pasta lunch energy intake (EI) $60 \mathrm{~min}$ after high-energy (HEP;607 kcal) and low-energy (LEP;246 kcal) preloads, and cumulative EI for the rest of the day, were measured in 15 overweight/obese volunteers (age:36.9 \pm 8.3years, BMI:31.3 $\pm 3.3 \mathrm{~kg} / \mathrm{m}^{2}$ ). Feelings of ap petite were assessed throughout using visual analogue scales. Fasting/ postprandial insulin, glucose and gut peptides plasma levels were also measured before and after the intervention.

Results: ANOVA showed a significant preload*exercise interaction ( $\mathrm{P}=$ 0.011 ) on cumulative EI and energy compensation over the same period improved significantly $(-87 \pm 196$ vs $68 \pm 165 \%, P=0.011)$. The same was not reflected acutely at the pasta lunch. ANOVA showed no effects of exercise, preload or interactions on feelings of appetite between preload and lunch. Weight loss was uncorrelated with improvements in energy compensation. A significant improvement in insulin sensitivity was observed.

Conclusion: This study suggests that exercise improves appetite regulation, over the course of a day, by leading to a more sensitive eating behaviour in response to previous EI in overweight/obese individuals The improvements observed seem to be independent of weight loss and a direct result of exercise itself. The improved appetite sensitivity may be related to increased insulin sensitivity; however, further mechanistic studies are needed.

Conflict of Interest: None disclosed. Funding: Martins C was supported by a Post Doctoral grant from FCT/FSE (Portugal) under the 3rd European Union community support programme.

\section{HT:PO.16}

The role of salivary PYY(3-36) in food intake and behavior - an alternative pathway for satiety

Acosta $A^{1}$, Voutetakis $A^{4}$, Aslanidi $G^{1}$, Geguchadze $R^{1}$, Doty $A^{1}$, Wright $A^{2}$, Campbell-Thompson $M^{2}$, Baum $B J^{4}$, Devine $D^{3}$, Zolotukhin $S^{1}$

${ }^{1}$ Division of Cellular and Molecular Therapy, Dept. Pediatrics, ${ }^{2}$ Dept. Pathology,

${ }^{3}$ Dept. Psychology, University of Florida, Gainesville, FI, USA

${ }^{4}$ Molecular Physiology and Therapeutics Branch, NIDCR, NIH Bethesda, MD, USA

Peptide YY (3-36) is a satiation gut hormone released postprandially by the gut. PYY(3-36) induces satiation by acting on Y2 receptors in the arcuate nucleus of the hypothalamus. Here we provide evidence for the existence of a novel alternative satiation pathway mediated by PYY(3 36). We found that PYY(3-36) is present in saliva and its concentration is correlated to its concentration in plasma. Also, PYY(3-36) and Y2 receptors are expressed in the taste cells in the circumvallate papilla of the tongue. In mice, the acute increase of salivary PYY(3-36) induces higher satiation showed by feeding behavioral studies and by c-Fos activation in the arcuate nucleus. This acute increase of salivary PYY(3-36) resulted in a decrease in one hour food intake in a dose-dependent manner. The chronic over-expression of salivary PYY(3-36) using a viral vector gene delivered (rAAV-PYY) into salivary glands produced a two-fold chronic 22 weeks increase of PYY(3-36) in saliva. This resulted in a significant decrease in weekly food intake and a $23 \%$ body weight loss 8 weeks after vector delivery compared to control. Interestingly, the chronic overexpression of salivary PYY(3-36) also produced an apparent reduction in aggressive behavior. This effect was tested in a blind study using a resident-intruder test. The treated mice showed a marked decrease in aggressive behavior compared with control mice. In conclusion, we have characterized a novel pathway for PYY(3-36) to induce satiation through saliva. We also found that the chronic over-expression of salivary PYY(3-36) decreases aggressive behavior in mice.

\section{HT:PO.17}

\section{Micronutrition-based therapy reduces obesity through a liver lipidome change in obese mice}

\section{El Kochairi I', Pradervand $S^{1}$, Lagrost $L^{2}$, Wahli $W^{\prime}$ \\ ${ }^{1}$ Center for Integrative Genomics, Lausanne, Switzerland ${ }^{2}$ INSERM Research Center UMR866, Dijon, France}

Introduction: The aim of this study is to investigate the effects of a specific micronutrients combination (Lipistase ${ }^{\circledR}$ ) on lipid metabolism and to identify the molecular mechanisms involved.

Methods: Two groups of genetically obese mice OB/OB were treated for 4 weeks with Lipistase ${ }^{\circledR}$, a combination of microdosed plant extracts that was incorporated in a standard diet or in a high fat diet. Control groups of sex and age matched $\mathrm{OB} / \mathrm{OB}$ mice were fed with the same diets without micronutrients. Plasma lipids, body weight, oxygen consumption, liver transcriptome and lipidome were analyzed.

Results: Treated animals gained significantly less weight versus controls when challenged with high fat diet. On the other hand, in the standard diet group, Lipistase ${ }^{\circledR}$ treated mice showed a significant decrease in plasma triglycerides and an increase of oxygen consumption compared to controls. In treated mice, the most upregulated gene in liver transcriptome was the fatty acid elongase (ELOVL6) that elongates palmitoleate to vaccinate and prevents hepatosteatosis and diet-induced obesity. Consistently, the vaccinate to palmitoleate ratio was significantly higher in the liver of treated animals versus controls.

Conclusion: This combination of micronutrients seems to enhance resistance to diet-induced obesity. Decreased plasma triglycerides and increased oxygen consumption further suggest a systemic effect of these micronutrients on lipid metabolism. We speculate that on the long term it may help preventing hepatosteatosis through ELOVL6 activation and subsequent liver fatty acid composition change. Finally, this is the first study showing that a combination of micronutrients may be a valuable therapeutic approach against obesity and lipid metabolism disorders.

Conflict of Interest: None. Funding: Research related to this abstract was funded by Bioresearch and Partners.

\section{HT:PO.18}

\section{Dietary protein and calcium have marked effect on weight} reduction induced changes in fatty liver metabolomics

\section{Pilvi $T K^{1,2}$, Korpela $R^{1,2}$, Finckenberg $P^{1}$, Orešič $M^{3}$, Mervaala $E M^{1}$ \\ ${ }^{1}$ Institute of Biomedicine, University of Helsinki, Finland ${ }^{2}$ Valio R and D Research Centre, Helsinki, Finland \\ ${ }^{3}$ VTT Technical Research Centre of Finland, Espoo, Finland}

Introduction: Fatty liver links obesity and insulin resistance. Weight loss is one of the few efficient ways to decrease liver fat. Both quantity and quality of lipids contributes to the pathophysiology of fatty liver. The amount of protein in the weight loss diet may effect the magnitude of weight loss, but the effect of protein source is not well characterised. In this study we show that the effect of weight loss on liver lipid and primary metabolite profile can be further improved by modification of dietary protein and calcium.

Methods: The liver metabolomic profile of lean and obese C57Bl/6J mice ( $\mathrm{n}=10$ /group) were compared with two groups of weight-reduced mice. Weight loss was achieved by energy restriction on control diet and whey 
protein-based high-calcium diet. The metabolomics analyses were performed using the UPLC/MS based lipidomics platform and the HPLC MS/MS based primary metabolite platform.

Results: Weight loss decreased the level of liver triacylglycerols, phospholipids and ceramides and increased the level of sphingomyelins, cholesterol esters and phosphatidylserines. Even significant weight loss did not restore the lipidomic profile to the level of lean animals. The effect of weight loss was significantly enhanced with high-calcium whey protein diet, which was also accompanied by marked increase of TCA cycle and poentose phosphate pathway metabolites in liver.

Conclusions: Weight loss induces marked changes in liver metabolic profile, but cannot restore the distribution of lipid species to the level of lean controls. The effect of weight loss is affected by the dietary protein source and calcium content.

Conflict of Interest: Pilvi TK and Korpela R are employees at Valio Ltd. Funding: The study was funded by Foundation for Nutrition Research, Finnish Funding Agency for Technology and Innovation and Valio Ltd.

\section{HT:PO.19}

\section{Differential inflammation gene expression in fat depots in} children

\author{
Tam $C S^{1,2,3}$, Heilbronn $L K^{3}$, Wong $M^{1,2}$, Cowell $C T^{1,2}$, \\ Cowley $M^{3}$, Kaplan $W^{\beta}$, Baur $L A^{1,2}$ \\ ${ }^{1}$ The Children's Hospital at Westmead, Sydney, Australia \\ ${ }^{2}$ Discipline of Paediatrics and Child Health, University of Sydney, \\ Sydney, Australia \\ ${ }^{3}$ The Garvan Institute of Medical Research, Sydney, Australia
}

Subcutaneous and visceral adipose tissues display metabolic, functiona and inflammatory differences, which may play a role in obesity-related low grade inflammation. The aim of this study was to identify differentially expressed inflammation genes between i) lean and overweight (owt)/obese (ob) and ii) subcutaneous and visceral fat in children.

Seventy-nine abdominal adipose biopsies (64 subcutaneous, 15 visceral) were obtained from children undergoing elective surgery ( 54 boys, 6.6 $\pm 5.4 \mathrm{yrs}, 29 \%$ owt/ob). Microarrays were performed on a subset of 20 samples. Inflammatory genes with $>2$ fold difference between subcutaneous and visceral fat were subsequently confirmed using RT-PCR in the whole cohort.

Interleukin (IL) 8 and 10, and chemokine ligand 2 mRNA levels were 6-, 4- and 2-fold higher in owt/ob children compared to lean children, respectively $(\mathrm{P}=0.04,0.02,0.03)$. IL-10, haptoglobin and IL-18 mRNA levels were 4-, 23- and 4-fold higher in visceral compared to subcutaneous fat $(\mathrm{P}<0.001,0.001,0.04)$. In contrast, neuronatin and vascular endothelial growth factor-d were 3 fold and 7-fold lower in visceral fat $(\mathrm{P}=0.03,0.01)$. There were no associations between gene expression and either BMI z score or HOMA-IR for any gene examined.

Obesity-associated low grade inflammation is established early in childhood. We found significant differences in expression of inflammatory genes in visceral compared to subcutaneous adipose tissue, supporting adult findings. We speculate that persistent levels of inflammation in obese children may develop to the low-grade inflammatory state characteristic of adult obesity.

Conflict of Interest: None disclosed. Funding: Diabetes Australia Research Trust 2007 grant. CST is funded by a National Health and Medical Research Council (NHMRC)/ National Heart Foundation Biomedical Postgraduate Scholarship and LKH is funded by a NHMRC Career Development Award.

\section{HT:PO.20}

Expression of the atrial natriuretic peptide and adrenergic receptor subtypes in human adipose tissue and adipocyte cultures

\section{Garruti $G^{1,2}$, Giusti $\mathcal{V}^{\beta}$, Nussberger $\mathcal{J}^{3}$, Darimont $C^{4}$, Appert-Collin $A^{2}$, Verdumo $C^{3}$, Nenniger Tosato $M^{2}$, Giorgino $R^{1}$, Giorgino $F^{1}$, Cotecchia $S^{2}$}

${ }^{1}$ Unit of Internal Medicine and Endocrinology, Department of Emergency and Organ Transplantations, University Hospital of Bari, Italy

${ }^{2}$ Department of Pharmacology and Toxicology, University of

Lausanne, Switzerland

${ }^{3}$ Department of Internal Medicine, Centre Hospitalier Universitaire

Vaudois, Lausanne, Switzerland

${ }^{4}$ Unit of Surgery and Liver Transplantations, Department of

Emergency and Organ Transplantations, University Hospital of Bari, Italy

${ }^{5}$ Nestlè Research Center, Lausanne, Switzerland

Introduction: Adrenergic receptors (AR)s and atrial natriuretic peptide (ANP) receptors (NP-R) are crucial for lipolysis and ANP inhibits the induction of inflammatory mediators by macrophages. Human pre-adipocytes express ANP and NP-R and secrete ANP.

Methods: The expression of AR subtypes, ANP and adipocyte differentiation markers (AdM) were analyzed by real-time RT-PCR in 3 models: subcutaneous (HSAT) and visceral (HVAT) fat biopsies, adipocyte primary cultures (hAdPrC) and a pre-adipocyte cell line (ChubS7) obtained from super-obese subjects.

Results: The expression of ANP, $\alpha_{1} A-A R$ and $\beta_{1}$-AR were comparable in HSAT and HVAT. The expression of $\alpha_{2} \mathrm{~A}-\mathrm{AR}$ and $\beta_{2}$-AR were higher in HSAT than in HVAT; $\beta_{3}$-AR were non-measurable in both fat depots. In both rosiglitazone-stimulated and non-stimulated hAdPrC and ChubS7 all ARs, except for $\beta_{3}$-AR, as well as ANP were expressed from day 3 to 6 of differentiation. In hAdPrC at day 7, ANP was already significantly lower than basally. After 17 days, in rosiglitazone-stimulated Chub-S7, ANP was switched off, AdM and $\beta_{3}$-AR switched on, $\beta_{2}$-AR increased and $\alpha_{2} \mathrm{~A}-\mathrm{AR}$ decreased as compared with basal.

Conclusions: Data on biopsies showing that both the pro-lipolytic $\beta 2$ $\mathrm{AR}$ and the anti-lipolytic $\alpha_{2} \mathrm{~A}-\mathrm{AR}$ are more expressed in HSAT than in HVAT suggest that the relative balance between these AR subtypes might be crucial in determining the amount of HSAT. Data on adipocyte cultures, providing evidence that $\beta_{3}$-AR appears when ANP disappears, indicate that at the end of the rosiglitazone-mediated differentiation adipocyte display $\beta_{1 / 2 / 3}$-AR-mediated lipolityc pathways but miss the autocrine/paracrine ANP-mediated pathway involved in lipolysis and inhibition of inflammation.

Conflict of Interest: None disclosed. Funding: Research relating to this abstract was funded by a Grant from Novartis Consumers' Health (to GG), Funds from the Italian Ministry of Health (to RG) and 'Fonds National Suisse de la Recherche Scientifique' (3100A0-100,703) (to SC).

\section{HT:PO.21}

In adult humans, the presence of UCP1 demonstrates that metabolically active cervical adipose tissue truly represents brown adipose tissue

Zingaretti $M C^{1}$, Crosta $F^{2}$, Vitali $A^{1}$, Guerrieri $M^{2}$, Frontini $A^{1}$ Cannon $B^{3}$, Nedergaard $\mathcal{J}^{3}$, Cinti $S^{1}$

${ }^{1}$ Department of Molecular Pathology and Innovative Therapies, Faculty of Medicine, Ospedali Riuniti Ancona, University of Ancona (Politecnica delle Marche), Italy

${ }^{2}$ Clinica di Chirurgia Generale e Metodologia Chirurgica, Ospedali Riuniti, Ancona, Italy

${ }^{3}$ Department of Physiology, The Wenner-Gren Institute, The Arrhenius Laboratories F3, Stockholm University, Sweden

Classically, adult humans have been considered not to possess active brown adipose tissue (BAT). However, positron emission tomography 
(PET) has shown, even in adults, fluorodeoxyglucose uptake that is distributed in such a way that it would seem to be BAT. Importantly, until now this interpretation has not been supported by direct evidence that these areas truly represented BAT, i.e. the presence of the BAT-unique protein UCP1. The PET images indicate that the root of the neck is a major potential BAT site. Therefore, to establish the presence of defined BAT in human adults, we obtained samples of subcutaneous peri-thyroid adipose tissue. These samples were removed from 35 patients undergoing surgery for different thyroid diseases. In a third of the samples, distinct areas composed of UCP1 immunoreactive brown adipocytes could clearly be discerned. In some cases, the brown adipocytes accounted for up to a third of all adipocytes. The brown adipose areas were richly innervated by sympathetic nerve fibers (visualised by tyrosine-hydroxylase immunoreactivity), clearly supporting that the tissue is under acute central control; adjacent white adipose areas did not demonstrate this feature The capillary density was high, implying a high capacity for delivery of oxygen. Remarkably, cells with features of brown adipocyte precursors were found in pericapillary areas. These data demonstrate that human adults are indeed provided with BAT and thus imply the possibilities of future therapeutic strategies for the treatment of obesity that include the activation of pre-existing brown adipocytes and particularly stimulation of the growth of pre-existing brown preadipocytes.

\section{HT:PO.22}

\section{Weight loss at 6 months with VI-0521 (PHEN/TPM combination) treatment}

Ryan $D^{1}$, Peterson $C^{2}$, Troupin $B^{2}$, Najarian $T^{3}$, Tam $P^{2}$, Day $W^{2}$ ${ }^{1}$ Pennington Biomedical Research Center, Baton Rouge, LA, USA ${ }^{2}$ VIVUS, Inc., Mountain View, CA, USA

${ }^{3}$ Los Ojos, CA, USA

Introduction: Previous studies have demonstrated that a $15 / 100 \mathrm{mg}$ combination of phentermine (PHEN) and controlled-release topiramate (TPM) induced greater weight loss than placebo or either of the single agent constituents of the combination. This phase 3,6 month, randomized, double-blind, placebo-controlled study evaluated the impact of 2 doses of VI- 0521 on body weight in generally healthy obese subjects. Methods: 756 subjects aged 18-71 years (mean 45.6 years) with BMI of 30-45 (mean 36.3) were randomly assigned to treatment with placebo, $7.5 / 46$ or $15 / 92 \mathrm{mg}$ PHEN/TPM, or single agent doses that comprise these combinations. All subjects received lifestyle and exercise guidance, and maintained a $500 \mathrm{kcal} /$ day deficit in caloric intake.

Results: Percent weight loss at 6 months for various PHEN/TPM doses are shown below:

\begin{tabular}{lllllllr}
\hline & Placebo & $7.5 / 0$ & $0 / 46$ & $7.5 / 46$ & $15 / 0$ & $0 / 92$ & $15 / 92$ \\
\hline Wt. loss (\%) & 1.71 & 5.45 & 5.13 & 8.46 & 6.06 & 6.44 & 9.21 \\
p-value vs: & & & & & & & \\
$\quad$ placebo & & & & $<0.0001$ & & & $<0.0001$ \\
PHEN & & & $<0.0001$ & & 0.0006 \\
TPM & & & & $<0.0001$ & & & $<0.0001$ \\
\hline
\end{tabular}

The percentage of subjects achieving at least $10 \%$ weight loss was $6.8 \%$ with placebo, $38.8 \%$ with the $7.5 / 46 \mathrm{mg}$ combination, and $40.8 \%$ with the $15 / 92 \mathrm{mg}$ combination. The most commonly observed adverse events included headache, paraesthesia, upper respiratory infection, dry mouth, nasopharyngitis, and constipation. No drug-related serious AEs were reported during the study.

Conclusion: Each of the 2 PHEN/TPM CR combinations evaluated in this study demonstrated significantly greater weight loss than both placebo, and the single-agent constituents that comprise the combination doses. Combinations of PHEN/TPM CR appear to be effective and well tolerated for the treatment of obesity.

Conflict of Interest: Ryan - VIVUS Scientific Advisory Board Member; Peterson, Troupin, Tam, Najarian, Day - VIVUS employee. Funding: No funding.

\section{HT:PO.23}

Tesofensine, a novel weight management drug, has no or minimal abuse potential

\section{Schoedel $K^{1}$, Meier $D^{2}$, Chakraborty $B^{1}$, Manniche $P^{2}$, Sellers $E^{1}$}

${ }^{1}$ Kendle Early Stage - Toronto, Toronto, ON, Canada

${ }^{2}$ NeuroSearch A/S, Ballerup, Denmark

Introduction: Tesofensine is a noradrenaline, dopamine and serotonin reuptake inhibitor in development for treatment of obesity. A Phase II study (24 weeks) showed highly significant and clinically relevant dosedependent weight reduction with tesofensine (TIPO-1) [Astrup A et al: Lancet 2008;372:1906-1913]. Because some appetite suppressants have stimulant properties, the purpose of this study was to thoroughly evaluate the abuse potential of tesofensine.

Methods: Single-dose, randomized, double-blind, crossover with 1, 6 and $9 \mathrm{mg}$ tesofensine versus placebo, 15 and $30 \mathrm{mg}$ dextroamphetamine (positive control), and $400 \mathrm{mg}$ bupropion/100 $\mathrm{mg}$ atomoxetine (negative controls $)$ in recreational stimulant users $(\mathrm{N}=52)$. Visual analogue scales, Addiction Research Center Inventory (ARCI) and other subjective measures were administered over $48 \mathrm{~h}$ and peak effects were analyzed by linear mixed model and t-test.

Results: Dextroamphetamine was significantly greater than placebo on all primary measures: Drug Liking, Overall Drug Liking, ARCI MBG ('Euphoria') and Amphetamine ('Stimulation'), and on all secondary measures, thereby confirming study validity. Tesofensine was not significantly different from placebo and was lower than dextroamphetamine $30 \mathrm{mg}$ on all primary measures. At some doses, tesofensine had lower Drug Liking than bupropion and lower Any/Good Effects and High than atomoxetine.

Conclusion: These results indicate that tesofensine has no or minimal abuse potential.

Conflict of interest: Authors are employees of Kendle Early Stage or NeuroSearch A/S. Funding: NeuroSearch A/S. 